\title{
La visita domiciliaria como estrategia para la atención primaria en familias de zonas rurales
}

\author{
The home visit as a strategy for primary care in families in rural areas
}

\section{A visita domiciliar como estratégia de atenção básica em famílias do meio rural}

\section{Angela Thalia Delgado Rojas'; Mirian Elena Saavedra Covarrubia²; Mirtha Flor Cervera Vallejos ${ }^{3}$; \& Rosa Jeuna Díaz Manchay ${ }^{4}$}

\footnotetext{
${ }^{1}$ Estudiante del X ciclo de la Escuela de enfermería. Universidad Católica Santo Toribio de Mogrovejo - Chiclayo, Perú. Orcid: https://orcid.org/0000-0001-7003-0318. Correo electrónico: pc1delgadorojas@gmail.com

${ }^{2}$ Doctor en ciencias de enfermería. Directora de la Escuela de Enfermería y Docente de la Universidad Católica Santo Toribio de Mogrovejo- Chiclayo, Perú. Orcid: https://orcid.org/0000-0002-8139-3816. Correo electrónico: msaavedra@usat.edu.pe

${ }^{3}$ Doctor en ciencias de enfermería. Especialista en nefrología. Docente de la Universidad Católica Santo Toribio de Mogrovejo- Chiclayo, Perú. Orcid: https://orcid.org/0000-0002-49721787. Correo electrónico: mcervera@usat.edu.pe.

${ }^{4}$ Doctor en ciencias de enfermería. Especialista en salud familiar y comunitaria. Docente de la Universidad Católica Santo Toribio de Mogrovejo- Chiclayo, Perú. Orcid: https://orcid.org/0000-0002-2333-7963. Correo electrónico: rdiaz@usat.edu.pe.
}

Correspondencia: Departamento de Ciencias de la Salud, Universidad Católica Santo Toribio de Mogrovejo, Av. San Josemaría Escrivá de Balaguer No 855, CP: 14001. Chiclayo, Lambayeque. Perú. Correo electrónico de contacto: rdiaz@usat.edu.pe

Para citar este artículo: Delgado Rojas, A.T., Saavedra Covarrubia, M.E., Cervera Vallejos M. F., \& Díaz Manchay, R. J. (2021). La visita domiciliaria como estrategia para la atención primaria en familias de zonas rurales. Cultura de los Cuidados, 25(61). Recuperado de http://dx.doi.org/10.14198/cuid.2021.61.11

Recibido:02/06/2021 Aceptado: 03/08/2021

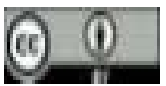

\section{RESUMEN}

Introducción: La visita domiciliaria, como estrategia educativa permite valorar a las familias en su contexto, esto influye en la promoción de la salud y la prevención de enfermedades. Aunque, existen limitaciones que generan deficiente intervención. Objetivo: Describir las experiencias del profesional de enfermería en la visita domiciliaria como estrategia para la atención primaria de la salud en las familias de zonas rurales. Métodos: Cualitativo con abordaje 
descriptivo, participaron 10 profesionales de enfermería que laboran en los centros de salud del área rural de Mórrope- Perú; el muestreo fue no probabilístico por conveniencia y la muestra obtenida por criterios de saturación y redundancia. Se empleó la entrevista semiestructurada con modalidad virtual a través de plataforma zoom y WhatsApp. Resultados: a) Motivos de la visita domiciliaria en la atención primaria: Identificación de riesgos, seguimiento y educación sanitaria, b) Estrategias que benefician la vista domiciliaria: Ganarse la confianza y atender las necesidades con sentido humanizado, c) Limitaciones para la ejecución de la visita domiciliaria: Creencias, inaccesibilidad, inseguridad y escasos recursos. Conclusiones: El profesional de enfermería, pese a las restricciones alinea su actuar a lo preventivo promocional, a fin de reconocer carencias y necesidades de la familia dentro de su entorno cultural.

Palabras clave: Visita domiciliaria; enfermería; promoción de la Salud; atención primaria de salud; investigación cualitativa.

\section{ABSTRACT}

Introduction: The home visit, as an educational strategy allows assessing families in their context, this influences health promotion and disease prevention. However, there are limitations that generate deficient intervention. Objective: To describe the experiences of the nursing professional in home visits as a strategy for primary health care in families in rural areas. Methods: Qualitative with descriptive approach, 10 nursing professionals working in health centers in the rural area of Morrope-Peru participated; the sampling was non-probabilistic by convenience and the sample was obtained by saturation and redundancy criteria. A semistructured interview was used with virtual modality through a zoom platform and WhatsApp. Results: a) Reasons for home visits in primary care: identification of risks, follow-up and health education, b) Strategies that benefit home visits: gaining trust and attending to needs with a humanized sense, c) Limitations for the execution of home visits: beliefs, inaccessibility, insecurity and scarce resources. Conclusions: The nursing professional, despite the restrictions, aligns his actions to the preventive-promotional, in order to recognize deficiencies and needs of the family within their cultural environment.

Keywords: Home visit; nursing; health promotion; primary health care; qualitative research.

\section{RESUMO}

Introdução: A visita ao domicílio, como estratégia educativa permite avaliar as famílias no seu contexto, isto influencia a promoção da saúde e a prevenção da doença. No entanto, existem limitações que geram uma intervenção deficiente. Objectivo: Descrever as experiências do profissional de enfermagem em visitas domiciliárias como uma estratégia para os cuidados de saúde primários nas famílias em zonas rurais. Métodos: Qualitativa com abordagem descritiva, participaram 10 profissionais de enfermagem que trabalham em centros de saúde na zona rural de Morrope-Peru; a amostragem foi não probabilística por conveniência e a amostra foi obtida por critérios de saturação e redundância. A entrevista semi-estruturada foi utilizada com modalidade virtual através da plataforma zoom e WhatsApp. Resultados: a) Razões para a visita ao domicílio nos cuidados primários: identificação de riscos, acompanhamento e educação sanitária, b) Estratégias que beneficiam a visita ao domicílio: ganhar confiança e satisfazer as necessidades com um sentido humanizado, c) Limitações para a execução da visita ao domicílio: crenças, 
inacessibilidade, insegurança e recursos escassos. Conclusões: O profissional de enfermagem, apesar das restrições, alinha a sua acção à preventiva-promocional, de modo a reconhecer as deficiências e necessidades da família dentro do seu ambiente cultural.

Palavras-chave: Visita ao domicílio; enfermagem; promoção da saúde; cuidados de saúde primários; investigação qualitativa.

\section{INTRODUCCIÓN}

La Visita domiciliaria (VD) está considerada como estrategia educativa, que emplea el profesional de enfermería (PE) para proporcionar la salud en el espacio íntimo de la persona como es el hogar; además le permite, detectar, evaluar, proteger y vigilar los problemas de salud existentes, en base a optimizar la calidad de vida, de manera individual o colectiva. Las actividades que se realizan son: valoración de necesidades, promover el autocuidado, promocionar estilos de vida saludable y el fomento del funcionamiento familiar ente otros aspectos (Sánchez \& Sarango, 2018). De este modo, la VD se convierte en un instrumento eficaz para mejorar y/o fortalecer la salud y bienestar de las personas en las diferentes etapas de vida (Parra, Gónzales \& Pinto, 2017). Por otro lado, la atención primaria de salud está basado en métodos, tecnologías y prácticas, para alcanzar y satisfacer las necesidades de las familias; sin embargo, la oferta de los servidos ha ido cambiando, con la finalidad de establecer respuestas a las expectativas del público y al número ilimitado de enfermedades. De ahí la importancia de la VD como estrategia, para proteger dichas necesidades en gran parte de la población, en especial las más vulnerables (Serrano, Carrillo, Santamaria, Cordero, \& Espinoza, 2018) como las personas, familias y comunidades de las zonas rurales.

A nivel de Latinoamérica, Colombia, la VD se realiza con el fin de disminuir los problemas actuales de la salud pública, donde, se construye la educación como una propuesta para orientarla y mejorarla. Así lo muestra la investigación (Hernández, 2017), al aludir que el PE educador constituye un componente fundamental en la formación de las familias, al conceder la libertad para tomar decisiones favorables sobre la salud. Sin embrago, tiene que lidiar con los recursos económicos y el bajo nivel educativo de las personas, transformándose en limitaciones condicionantes para no lograr una intervención exitosa.

Por su parte, la investigación de Guevara \& Solis (2017), mencionan que la estrategia que humaniza la atención a las necesidades es la VD, la cual accede el ingreso 


\section{4}

a los servicios de salud a través de la educación impartida en las escuelas, el trabajo y el hogar; donde el PE identifica de manera oportuna los riesgos que afectan la salud de todas estas colectividades. Se suma (Mendoza \& Abarca, 2017), al argumentar que las VD no se ejecutan en más del 50\%, lo que constituye una dificultad para el éxito de esta estrategia; puesto que, al realizarla se identifica los riegos existentes que generan inseguridad en la salud de las personas y a través del seguimiento continuo y oportuno, se logra mejorar las condiciones de vida. De modo que, la cantidad de visitas contabilizadas no cubre la cobertura poblacional estimada y no genera impacto en las interacciones sensibles de la atención básica (Leite, Sacramento, Oliveira \& Ferreira, 2019).

Por ello, resulta importante la VD que realiza el personal de enfermería en la comunidad rural de Mórrope, esta pertenece al departamento de Lambayeque en Perú, es una zona turística por su bagaje cultural y tienen muy arraigadas algunas creencias y costumbres que pueden influir en su salud. En ese sentido, el PE realiza VD como una estrategia preventiva promocional, con la finalidad de realizar seguimiento a las familias, a través de las diversas estrategias sanitarias que se desarrollan en los servicios de salud del primer nivel de atención. Estas visitas son realizadas cuando: hay inasistencia a la consulta programada, abandono del tratamiento, etc. Por ello, se considera significativa profundizar en esta temática, porque el trabajo que realiza el PE en atención primaria le permite mejorar las coberturas de las estrategias sanitarias y por consiguiente contribuir a mejorar los indicadores de salud y a disminuir la morbimortalidad en el área sociosanitario correspondiente. Con ello, surgió los objetivos: Describir las experiencias del profesional de enfermería en la visita domiciliaria como estrategia para la atención primaria de la salud en las familias de zonas rurales.

\section{MÉTODO}

Participantes: Investigación de enfoque cualitativo (Hernández, 2018), con abordaje metodológico descriptivo (Berenguera, y otros, 2014). La población estuvo conformada por 32 profesionales de enfermería. El muestreo se constituyó por conveniencia, obtenida por la técnica de saturación y redundancia, al llegar a 10 profesionales de enfermería de ambos sexos, que laboran en los puestos de salud del primer nivel de atención de Mórrope en Lambayeque, Perú. En su mayoría eran personal nombrado, edades comprendidas entre 30 a 64 años, con experiencia profesional entre 6 
y 25 años, tenían estudios de especialidad en salud familiar y comunitaria, pediatría, crecimiento y desarrollo, administración, oncología, nefrología, y solo dos tuvieron maestría en salud pública.

Instrumento: Para recolectar los datos, se empleó la técnica de entrevista semiestructurada, con modalidad virtual a través de plataforma zoom, WhatsApp y vía telefónica, la cual constó de un título, datos generales y de 6 preguntas abiertas. El instrumento fue validado a través de 4 juicios de expertos. Después tuvo el pase del Comité de Ética en Investigación de la Facultad de Medicina, de la Universidad Católica Santo Toribio de Mogrovejo mediante resolución N 297-2020-USAT- FMED, luego se realizó la prueba piloto con dos enfermeras que no formaron parte de la muestra.

Las entrevistas se realizaron una por día previo consentimiento informado, las mismas que fueron transcritas de inmediato y al plazo de tres días, se presentó a cada entrevistado de forma virtual (WhatsApp), para que verifiquen los datos emitidos sobre sus experiencias en visita domiciliaria como estrategia para la atención primaria en familias de zonas rurales.

Procedimiento: Alcanzado el permiso de la Gerencia Regional de Salud y de los jefes de centros y puestos de salud de Mórrope; se ubicó a los profesionales de enfermería que realizan las VD, por vía virtual WhatsApp y llamada telefónica dada la situación de pandemia por COVID-19 que afronta el estado peruano e informarles los objetivos del estudio, obtenida su participación con precedente de consentimiento informado, se acordó día y hora de entrevista vía Zoom según su disponibilidad. Las preguntas fueron concisas y realizadas en un tiempo de 20 y 30 minutos. La identidad de los participantes se protegió con el nombre de piedras preciosas así la primera fue perla y el último zafiro, de esta forma fue posible identificarlos al procesar los datos.

Entre las consideraciones éticas (Sgreccia, 2014) : Se respetó en todo momento la autonomía de los entrevistados, por lo cual se otorgó vía virtual ( Zoom, WhatsApp) y vía oral a través de la llamada telefónica, el consentimiento informado con un lenguaje claro y sencillo para conseguir de manera voluntaria la aceptación verbal; asimismo, se dio a conocer que la información obtenida fue confidencial y solo utilizada para fines académicos, con la seguridad de que en ningún momento se perjudicó su bienestar ni hubo ningún tipo de daño. Como criterios de rigor científico asumidos estuvieron: La credibilidad, audibilidad y transferibilidad (Berenguera, y otros, 2014). En el estudio 
convergieron datos reales y verdaderos, con exactitud en las transcripciones de las entrevistas de los participantes sin tergiversar datos, luego de trascritos fueron devueltos a cada participante vía WhatsApp para su verificación.

Análisis de los datos cualitativos: Los datos adquiridos fueron procesados por análisis de contenido (Hernández, 2018), en tres etapas: a) Pre-análisis, permitió escuchar hasta tres veces las grabaciones de voz, luego revisar, organizar y seleccionar los datos con ideas más relevantes según las experiencias de cada enfermera, bibliografía previa y práctica de las investigadoras. b) La codificación, se transcribieron las grabaciones a Word sin modificar el significado; luego se realizó una matriz de categorización, para obtener datos útiles, se agruparon según significado en relación con el objeto de estudio, a estos se les asigno códigos y los datos más importantes se convirtieron en unidades de análisis por similitud o divergencia. C) La categorización; se procesó en tablas, las categorías centrales, los códigos, y las citas literales de las entrevistas, luego para terminar se acoplaron los discursos más explicativos de los enfermeros, para llegar a una comprensión de las categorías, después se analizó y discutió con bibliografía científica.

\section{RESULTADOS}

a) Motivos de la visita domiciliaria en la atención primaria: Identificación de riesgos, seguimiento y educación sanitaria

La VD involucrada en el primer nivel de atención, conforma la puerta de entrada al sistema de salud, por esta razón es con frecuencia la actividad primordial del personal de salud; para: informar, educar, evaluar condiciones socio-económico, promoverprevenir enfermedades y establecer acuerdos con las familias (Sánchez \& Sarango, 2018). Tal como lo afirman en las siguientes las declaraciones:

"La VD se realiza en el primer nivel con la finalidad de conocer los problemas, las vulnerabilidades, las carencias de la persona, familia o comunidad... sirve para encontrar factores de riesgo" (Perla).

"La VD es el núcleo de la promoción de la salud para conocer a la población y aplicar los diferentes paquetes de atención integral de acuerdo a la etapa de vida..." (Topacio)

En este contexto, realizan la VD para hacer seguimiento al cumplimiento del calendario de vacunación, control de crecimiento y desarrollo (CRED), monitorizar la 
adherencia de los niños que reciben suplementación de hierro, evidenciándose en los siguientes discursos:

"Cuando llega un niño al consultorio para su control de CRED y se indica suplementación de hierro preventivo; se realiza VD para verificar la adherencia del hierro, y educar a la madre..." (Ámbar).

"Realizamos el seguimiento a los niños con anemia, a los que están atrasados en su calendario de vacunación, tienen desnutrición o no van al consultorio de CRED..." (Turquesa).

De igual forma, algunas visitas domiciliarias, tienen la peculiaridad de ser realizada por un equipo completo de salud, lo cual, depende de la disponibilidad del recurso humano tal como lo declaran:

"actividad donde el personal de salud (técnico, enfermera, obstetricia u otro profesional) llega a la casa de la familia en la comunidad con los objetivos preventivos y brindar información” (Diamante).

b) Estrategias que benefician la vista domiciliaria: Ganarse la confianza y atender las necesidades con sentido humanizado

En la VD, el PE se "gana” a la familia, para impartir confianza, seguridad y conocer a la familia, para atender sus necesidades con sentido humano. Como se evidencia a continuación:

"Se tiene que conocer la población e integrarse en la comunidad con liderazgo, y tener su confianza para que los integrantes te digan que está pasando en su familia...” (Diamante).

"Es importante la primera impresión que tiene de ti la familia... siempre hay que ser muy amable saludando y presentándose, explicando cuál es tu finalidad, así te dejan pasar y te brindan toda su confianza" (Topacio).

“Tener una población accesible con tiempo disponible, es lo ideal, porque las personas te van conociendo, se muestran abiertas a recibir educación... todo depende de como nos familiarizarnos y adecuarnos a ellos, porque al ver la realidad buscas la forma que eviten las enfermedades” (Perla). 
Los PE refieren que en la VD después de ganarse la confianza de la familia, se inicia una comunicación abierta sobre sus necesidades, las vulnerabilidades de los integrantes que viven dentro de su contexto, de esta manera, se pueda planificar cuidados integrales y humanizados de acuerdo a su realidad y los recursos con que disponen las familias, y por medio de la educación en salud, sean los mismos individuos los que cuidan de su propia salud, evitando así complicaciones.

\section{c) Limitaciones para la ejecución de la visita domiciliaria: Creencias, inaccesibilidad, inseguridad y escasos recursos}

El PE, muchas veces tienen que lidiar con algunas limitaciones como las creencias bien arraigadas de las familias, el difícil acceso a los hogares, los peligros e inseguridades en las calles, unido a los escasos recursos materiales y humanos para que cumplan adecuadamente las VD. Reconocen que trabajar con la idiosincrasia de las familias, es difícil, como lo expresan en los siguientes discursos:

“Las personas a las que visitamos son reacias, nos cierran la puerta... Si vamos muy temprano o tarde y estan ocupados, no nos hacen caso....se dejan llevar por lo que dice la vecina, en todo Mórrope lo tienen bien arraigado...De repente las señoras utilizan algodón en la cabecita del recién nacido, y no podmeos eviatarles eso, sino debemos adecuarnos a su cultura” (Diamante).

"Para encontrar a la mamá de un niño, es a través de los suegros; por lo cual, es ir conocimiento a la población, cuál es su idiosincrasia cultural para poder trabajar con ellos... pero, no consideran importante el control de CRED, las vacunas, el descarte de anemia; además cuando tienen una persona delicada, no lo llevan al centro de salud sino al curandero" (Perla).

Otra dificultad manifestada es la inaccesibilidad para hacer las VD, ya sea porque las casas están ubicadas en zonas muy lejanas, el clima es otro impedimento por el calor excesivo. Unido a los riesgos y accidentes a los que tienen que enfrentar, evidenciado en los siguientes discursos:

"Tengo colegas que fueron mordidas por los perros callejeros, otra fue víctima de intento de violación, por eso es importante conocer la población...es difícil salir a caminar sola...” (Topacio).

"Quedan muy alejadas las casas, aunque hay moto pero cobran caro y gatamos de nuestro dinero porque el MINSA no cubre la movilidad... en verano el calor es infernal...” (Zafiro). 
Otra de las dificultades es la escasez de recursos materiales y humanos para realizar VD:

"Nuestra principal dificultad y limitación es la alta demanda de pacientes y la gran brecha de personal que tenemos para comenzar a realizar la VD, por eso no las podemos hacer continuamente... no hay presupuesto para los pasajes...” (Ámbar) "Hay escases de recursos humanos, lo cual no te permite realizar la VD, al menos nosotros que somos un puesto, contamos con un personal técnico que muchas veces está haciendo las labores de admisión, de triaje y no podemos nosotros salir...” (Zafiro).

\section{DISCUSIÓN}

En este estudio los profesionales de enfermería, explican que es fundamental la identificación de los recursos, la prevención de riesgo y el trato directo con las familias por medio de la VD, ya que es la familia quien conoce las carencias, necesidades, problemas y anhelos. Como lo manifiestan los estudios de Stockert \& Hall (2019) y Ishikawa, Fujiwara \& Nakayama (2015), quienes afirman que la VD en la atención primaria de salud permite conocer de primera línea las necesidades y los recursos con los que disponen las familias. Dicho conocimiento les permite orientar, ayudar y prevenir problemas que les genera daño y tener la posibilidad de prevenir complicaciones a futuro a fin de minimizar gastos innecesarios; por ello, adecuan estrategias como el plan de intervención, para que actúen sobre el problema identificado, impidiendo que llegue a niveles mayores. Los PE realizan la VD para hacer seguimiento cuando el niño no está asistiendo a sus vacunas, controles de crecimiento y desarrollo y para establecer acuerdos con las madres de niños que están recibiendo suplementación de hierro o tienen desnutrición. Al respecto, Vega et al. (2020) considera necesario mejorar el seguimiento de los niños desnutridos visitando sus casas para educar a la madre y según sus recursos se le enseñe a preparar dietas equilibradas. Veramendi y Soto (2018), indicaron que, en la VD las madres conocieron los alimentos ricos en hierro, la elección, combinación y conservación del suplemento antes de su preparación. Sin embargo, el estudio de Anto et al (2019) difiere, porque la baja cobertura de VD, originó que la mayoría de niños resulte con anemia leve.

En ocasiones las VD, se realiza por el equipo interdisciplinario con el propósito de prevenir e informar a los integrantes de la familia, quienes gozan de buena salud o se encuentren en alto riesgo y por su misma naturaleza de vulnerabilidad, tienen que ser 


\section{0}

intervenidos. Esto guarda relación con Sánchez, Betancurth \& Jimenez (2020), al exponer que la VD da la oportunidad de comprender realidades concretas de la salud; además, enfatizan que se debe contar con un equipo interdisciplinario que permita un acompañamiento integral a las familias, destacando la presencia del PE como agente de cuidado y como guía en la realización.

Por otro lado, este estudio refleja algunas estrategias que son benéficas para la VD, dentro de ellas esta el liderazgo, los conocimientos, la amabilidad, la seguridad y la confianza, para que sea más fácil llegar a la población y ganarse la confianza de la familia con la cual se programa trabajar para brindar un cuidado integral y humano. Específicamente, el liderazgo en las VD, permite al PE sumergirse en su realidad como un líder y buscar oportunamente la manera de prevenir enfermedades; calificaciones que coinciden con Herrera, Chamorro \& Costilla (2018), al argumentar que se debe reconocer el liderazgo del PE, pues constituyen el vínculo entre la comunidad y los servicios de salud.

Los PE sustentan, que es importante conocer el tiempo que disponen las familias, lo que les permite lograr los objetivos de la VD, además tienen que adecuarse a las necesidades y/o problemas identificados, y por medio de la educación en salud, sean los mismos individuos los que cuiden de su propia salud, evitando así complicaciones. Resultados semejantes a los aportes de Geroy, Fernández, et al (2019), cuando señalan que el PE, planea y organiza su trabajo según las necesidades del grupo etario y se encargan de reconocer problemas de salud familiar y efectuar acciones de intervención con miras a indagar su solución, con la cooperación de los miembros del grupo familiar.

Asimismo, recalcan los PE, que es importante la manera de presentarse, el cual debe ser con amabilidad, lo que genera un clima confianza y respeto, con el propósito de resolver las inquietudes e interrogantes de la familia en la VD. Por su parte, el Ministerio de Salud (2014), da a conocer que el desarrollo de la VD es de manera confidencial, dirigida a fomentar la participación de la familia en un clima de confianza y respeto, y con delicadeza solicitar el ingreso al domicilio. Vega-Ayasta, et al. (2020), concluye que las enfermeras cuidan con amabilidad, pues practican la cortesía, esto genera confianza tanto en el paciente como en la familia. Costa et al (2020), sugiere a los trabajadores sanitarios de atención primaria que durante las visitas a domicilio promuevan y refuercen los vínculos con los usuarios. Para Guevara \& Solis (2017), la VD constituye una 
herramienta que humaniza el cuidado, porque democratiza la salud, al brindar atención universal independientemente de la condición de paciente y los accesos a los centros de salud.

Asimismo, los PE manifiestan que las personas tienen bien arraigado su cultura, lo cual, lo exteriorizan en la forma de cuidarse, utilizando su saber popular de sus ancestros, por ello, tienen que adecuar estrategias para que trabajen en base a su cultura y no prohibirles a realizar estas acciones. Al respecto, Bustamante et al (2019), indican que a pesar que el personal de salud eduque a la madre sobre cuidados del niño en el hogar, cuando realizan las VD se percatan que más prevalece sus prácticas culturales. De esta manera, se da a conocer que cada grupo o subgrupo comprende sus propias prácticas, por lo que resulta imposible homogenizar el cuidado.

Mencionan los PE que enfrentan dificultades para que ejecutar la VD y no las realizan, tales como el escaso recurso de personal y la alta demanda de pacientes; a pesar de ello, se enfocan por cumplirlo. En ese sentido, Savassi (2016), concluye que la atención domiciliaria sigue siendo descuidada y amenazada por una demanda de atención que supera la capacidad asistencial de los equipos. Por ello, la OPS (2019), recomienda con carácter de urgencia, la necesitada de extender el número de PE en el primer nivel de atención, para apartar obstáculos de acceso hacia la salud y ampliar una gama de cuidados en áreas con escaso personal sanitario. Asimismo, el MINSA (2016), indica que la organización de los establecimientos de salud responde limitadamente a las necesidades y demandas de la población, todos los niveles de atención son deficientes en infraestructura y financiamiento.

Los PE también declaran que no cuentan con los recursos para realizar la VD, porque realizarla demanda tener la implementación para trasladarse a las zonas más alejadas, por ello optan por ir en mototaxi, pero les cobran caro y esos gastos muchas veces no son reconocidos por el establecimiento de salud y tienen que realizarlo con sus propios recursos, esto se debe porque en la actualidad estos programas no vienen presupuestados. Resultados similares al estudio de Puch \& Jara (2015), reporta que existen familias que viven alejadas de los diversos centros de salud, por lo que tomar movilidad y tener la implementación adecuada constituye un recurso y gasto adicional con lo que los profesionales no cuentan. 
Asimismo, los PE exteriorizan que han sufrido accidentes al ejecutar la VD, como la mordedura de un perro, caídas y perderse en la zona, e incluso intento de violación. El estudio de Muñoz, Gonzales, Sánchez, \& Silva (2020) explica que el PE se enfrenta al salir del puesto de salud a la delincuencia y agresividad de las personas, lo que le dificulta realizar la visita. Otro estudio refiere que al realizar VD se pueden encontrar calles con inseguridad, marginación, tener accidentes e incluso de ser agredidos (Morales, 2018).

\section{CONCLUSIONES}

La experiencia de los profesionales de enfermería que ejecutan la VD a las familias como estrategia para la atención primaria en Mórrope, alinean su actuar a lo preventivo promocional, con la finalidad de reconocer sus carencias y necesidades dentro de su entorno natural para reducir factores de riesgo de manera oportuna, impidiendo a futuro que se complique la salud de las personas. Para ello educan en salud queriendo mejorar la calidad de vida a nivel individual y colectivo.

Los profesionales de enfermería como parte de su experiencia al realizar la VD, destacan como una cualidad indispensable el liderazgo para proporcionar seguridad al interactuar con la familia, para optimizar y promover la calidad de vida de las personas vulnerables, de manera confidencial en una atmosfera de confianza y respeto. Además, consideran a la VD como una estrategia que humaniza la atención de las necesidades de manera universal, porque permite el ingreso a los espacios privilegiados donde se desenvuelve de manera espontánea la persona, familia y comunidad.

Los profesionales de enfermería que realizaron la VD, experimentaron diversas restricciones como la cultura de la población que influye significativamente, al encontrar otros estilos de vida, por lo que reorientan su trabajo en concientizar y modular aspectos negativos que ponen en peligro la salud, además la falta de recursos económicos les impide trasladarse a zonas más alejadas generando atraso en reconocer de primera fuente la problemática existente; sumado a ello, la alta demanda de pacientes y el escaso recurso del personal de salud, genera gran preocupación ya que muchas veces en la zona urbanorural la visita domiciliaria no es ejecutada, por no contar con la disponibilidad para el desplazamiento y por contar con escaso personal de enfermería.

Conflicto de intereses: Los autores declaran no tener conflictos de intereses.

Financiamiento: Ninguno. 


\section{BIBLIOGRAFÍA}

o Anto, J., Nicho, M. Castañeda, A., Casas, B., \& Miranda, N. (2019). Anemia y visita domiciliaria en niños atenidos en establecimiento el primer nivel de Lima norte. Health Care \& Global Health. 3(1), 29-33. doi: https://doi.org/10.22258/hgh.2019.31.52.

o Berenguera, A., Fernández, S., Pons, M., Pujol, E., Rodríguez, D., \& Saura, S. (2014). Escuchar, Observar y comprender. Recuperando la narrativa en las ciencias de la salud. Aportaciones de la investigación cualitativa. Barcelona: Institut Universitari d’Investigació en Atenció Primària Jordi Gol.

o Bustamante Llatas, J., Gordillo Julón, M., Díaz Manchay, R., Mogollón Torres, F., Vega Ramírez, A., \& Tejada Muñoz, S. (2019). Lactancia materna, alimentación complementaria y suplementación con multimicronutrientes: Perspectiva intercultural. Cultura de los cuidados, 23(54), 231-243. doi: https://doi.org/10.14198/cuid.2019.54.20

o Costa D., de Almeida A., Mara CL., de BritoT, Fernandes M., et al. (2020). Satisfação do usuário da Atenção Primária no Distrito Federal: a importância do acesso oportuno e da visita domiciliar. Ciênc. saúde coletiva 25 (5) 08. Recuperado dehttps://www.scielosp.org/pdf/csc/2020.v25n5/1851-1863/pt

o Geroy, A., Fernández, A., Núñez, A., \& López, T. (2019). La visita integral a la familia. Guía Metodológica para su ejecución. Electrónica: Medi Sur, 5. Recuperado de http://medisur.sld.cu/index.php/medisur/article/view/4406/2968 $\underline{75}$

o Guevara, G., \& Solis, K. (2017). Visita Domiciliaria: Un espacio para la adquisición y modificación de prácticas en salud. Enfermería actual de Costa Rica, 14. Recuperado de https://www.scielo.sa.cr/pdf/enfermeria/n34/1409-4568enfermeria-34-82.pdf

o Hernández R, M. C. (2018). Metodología de la investigación las rutas cualitativas, cualitativa y mixta. México: Mc Graw Hill.

o Hernández, L. (2017). El rol educativo del profesional de enfermería en los programas de promoción, prevención y tratamiento de salud. Corporación universitaria adventista, $109 . \quad$ Recuperado de http://repository.unac.edu.co/bitstream/handle/11254/504/Proyecto\%20de\%20G rado.pdf?sequence $=1 \&$ is Allowed $=y$

o Herrera, M., Chamorro, E., \& Costilla, T. (2018). Enfermería de salud comunitaria: Promoción de conductas saludables en una comunidad rural del 
Estado de México. Enferm Inst Mex Seguro Soc, 8. Recuperado de https://www.medigraphic.com/cgi-bin/new/resumen.cgi?IDARTICULO=80657

o Ichikawa, K., Fujiwara, T., \& Nakayama, T. (2015). Effectiveness of Home Visits in Pregnancy as a Public Health Measure to Improve Birth Outcomes. PLOS ONE, 4. doi: https://doi.org/10.1371/journal.pone.0137307

o Leite, M., Sacramento, D., Oliveira, H., \& Ferreira, M. (2019). Home visits of the nurse and its relationship with hospitalization by diseases sensitive to primary health care. Esc Anna Nery 2019;23(2),e20180285. doi: 10.1590/2177-9465EAN-2018-0285.

o Mendoza, N., \& Abarca, A. (2017). Eficacia del Monitoreo de la Visita Domiciliaria Efectuada por Enfermeras a Niños Menores de Tres Años Suplementados con Multimicronutrientes del Centro de Salud Alto Selva Alegre Arequipa 2016. Universidad Católica de Santa María, 77. Recuperado de http://tesis.ucsm.edu.pe/repositorio/handle/UCSM/6616

o MINSA (2014). Manual de visitas domiciliarias mirando y acompañando con cuidado y afecto. Proyecto aprendiendo ayudando, 41. Recuperado de https://www.mimp.gob.pe/files/direcciones/dgnna/manual-de-visitasdomiciliarias-FINAL.pdf.

o MINSA (2016). Programa nacional de formación en salud familiar y comunitaria (PROFAM). 201. Recuperado de https://cdn.www.gob.pe/uploads/document/file/344297/Programa_nacional_de_f ormaci\%C3\%B3n_en_salud_familiar_y_comunitaria20190725-19981-

1pm58a2.pdf.

o Morales, R. M. (2018). Experiencias de las enfermeras al brindar cuidados integrales en el primer nivel de atención. ACC CIETNA rev la Esc Enfermería, 1, 33-40. doi: https://doi.org/10.35383/cietna.v1i1.162

o Muñoz, O., Gonzales, C., Sánchez, K., \& Silva, N. (2020). Factores asociados al cumplimiento de las visitas domiciliarias por parte del equipo sanitario del centro de salud del MSP de la ciudad de Guayaquil. Journal of business and antrepreneurial $\quad$ studies, $\quad$ Recuperado de https://dialnet.unirioja.es/servlet/articulo?codigo=7472725

o OPS. (2018). OPS insta a ampliar el rol de las enfermeras en la atención primaria de salud. Recuperado de https://www3.paho.org/hq/index.php?option=com.

o Parra, G., Gónzales, M., \& Pinto, G. (2017). Proceso de Enfermería en un estudio de familia de persona adulta mayor en atención primaria de salud. Enfermería Universitaria, 16. 14(1). doi: https://doi.org/10.1016/J.REU.2016.11.003

o Puchia, C., \& Jara, P. (2015). Enfermería y el cuidado domiciliario de los mayores en la era de la globalización. Enfermería Universitaria,12(4), 219-225. 
o Sánchez, J., \& Sarango, W. (2018). Rol del profesional de enfermería en atención primaria de salud en las unidades operativas del distrito 11D01 - Zona 7. Universidad Nacional de Loja, 129p. Recuperado de https://dspace.unl.edu.ec/jspui/handle/123456789/20647

o Sánchez, N. (2020). La visita familiar desde los determinantes sociales de la salud: aporte de enfermería a la atención primaria. Cuidarte, 11, 4. doi: https://doi.org/10.15649/cuidarte.935

o Serrano, C., Carrillo, T., Santamaria, W., Cordero, I., \& Espinoza, K. (2018). Metodología para el pase de visita de Enfermería en la atención primaria de salud. Medica Electrónica, $10 . \quad$ Recuperado de http://www.revmedicaelectronica.sld.cu/index.php/rme/article/view/2276

o Sgreccia, E. (2014). Manual de Bioética. La bioética y sus principios (4a ed.). Madrid:BAC-Maior.

o Souza, S., Ferreira, A., Aguilar, R., Veloso, O., \& Vieira, M. (2013). El liderazgo en la visión de Enfermeros Líderes. Enfermería Global, 12(30), 268-280.

o Stockert, P., \& Hall, P. (2019). Fundamentos de Enfermería: Práctica de la Enfermería basada en la comunidad. Barcelona: Elsevier.

o Vega-Ayasta, M. T., Díaz-Manchay, R. J., Cervera-Vallejos M. F., RodríguezCruz, L. D., Tejada-Muñoz, S., Guerrero-Quiroz, S. E. (2020). Amabilidad, confort y espiritualidad en los cuidados paliativos oncológicos: Aporte para la humanización en salud. Cultura de los Cuidados, 24 (58).doi: http://dx.doi.org/10.14198/cuid.2020.58.05

o Vega Ramírez, A., Barragán Piñin, J., Torres Torres, K., Díaz Manchay, R., Mogollón Torres, F., \& Tejada Muñoz, S. (2020). Alimentación en el contexto familiar y escolar del niño con desnutrición de una zona rural. Cultura de los cuidados, 24(56), 167-182. doi:https://doi.org/10.14198/cuid.2020.56.12

o Veramendi, N., \& Soto, J. (2019). Intervención de la enfermería un abordaje educativo en prevención de la anemia. Investigación y Postgrado, Vol. 34(1), 5977. 